\title{
Coherent distributions for the rigid rotator
}

\author{
Marius Grigorescu
}

This work presents Wigner-type quasiprobability distributions for the rigid rotator which become, in the limit $\hbar \rightarrow 0$, coherent solutions of the classical Liouville equation known as the "action waves" of Hamilton-Jacobi theory. The results are consistent with the usual quantization of the intrinsic angular momentum, but for the expectation value of the Hamiltonian, a finite "zero point" energy term is obtained. It is shown that during the time when a quasiprobability distribution evolves according to the Liouville equation, the related quantum wave function should satisfy the time-dependent Schrödinger equation. 


\section{Introduction}

The models of rigid rotation concern finite many-particle systems having a well-defined instantaneous intrinsic frame. The space $Q$ of static configurations is in this case the group $S O(3, \mathbb{R})$ while the low-energy dynamics can be described as a Hamiltonian flow on the phase-space $M=T^{*} S O(3, \mathbb{R})[1]$.

For the atomic nuclei such an intrinsic frame is defined by a deformed mean-field. The residual interactions between protons and neutrons can also be taken into account by models of two coupled rotators, applied to study the low-lying isovector magnetic excitations [2, 3]. Similar considerations for the isospin degree of freedom have been presented in [4, 5].

Distributions on $T^{*} S O(3, \mathbb{R})$ appear in the treatment of statistical ensembles of microscopic rotators. Such ensembles are particularly interesting because unlike the space $Q=\mathbb{R}^{3}$ of the translation coordinates, the spaces $S O(3, \mathbb{R})$ of the intrinsic rotations are physically distinct and of finite volume $\left(v_{R}=8 \pi^{2}\right)$. For the polyatomic molecules the moments of inertia are large, and although the statistical weight may include the nuclear spin degeneracy [6], the thermal equilibrium is described by the classical Boltzmann distribution. At low temperatures the partition function is calculated using the spectrum of the quantum Hamiltonian, which is well-known up to a controversial "zero-point" energy term. Proposed during the early days of the quantum theory [7] to explain the specific heats of diatomic gases, finite ground state-energy terms have been retrieved in the more recent years for the rigid rotator from geometrical considerations [8, 9, 10], and for the simple (linear) rotator by using reduced Wigner quasiprobability distributions [11]. Though, by adapting the known Wigner transform $\left[12\right.$ from $\mathbb{R}^{3}$ to $S O(3, \mathbb{R})$, an additional "quantum potential" term was obtained [13].

In this work Wigner-type distributions for the rigid rotator will be introduced along the lines of [14, 15, 16], by discretizing the Fourier transform in momentum of the "action waves" on $T^{*} S O(3, \mathbb{R})$. The classical dynamics of the rigid rotator is presented in Section 2, both as a Hamiltonian system on $T^{*} S O(3, \mathbb{R})$ and as geodesic motion on $S O(3, \mathbb{R})$. The coherent solutions of the classical Liouville equation provided by the "action waves" of the Hamilton-Jacobi theory are presented in Section 3. It is shown that by a suitable discretization of their Fourier transform in angular momentum

\footnotetext{
${ }^{1}$ The term "rotator" was preferred here for being closer to "rotating body" than the common form "rotor". Though, there are references in which "rotator" designates only a linear rotating object $\left(I_{1}=I_{2}>0, I_{3}=0\right)$.
} 
it is possible to obtain quasiprobability distributions on $T^{*} S O(3, \mathbb{R})$ which are consistent with the usual quantum treatment. However, for the expectation value of the Hamiltonian, a finite "zero point" energy is obtained. Conclusions are summarized in Section 4.

\section{The Euler equations}

The group $S O(3, \mathbb{R})$ consists of the $3 \times 3$ real, orthogonal, unimodular matrices,

$$
S O(3, \mathbb{R})=\left\{\mathcal{R} ; \mathcal{R}^{T} \mathcal{R}=I, \operatorname{det} \mathcal{R}=1\right\} .
$$

This group is a compact manifold, such that every rotation $\mathcal{R}$ can be specified by the versor $\mathbf{g}$ of the rotation axis and the angle $\gamma$ of rotation around this axis, which means by the vector $\gamma \mathbf{g} \in \mathbb{R}^{3},|\mathbf{g}|=1, \gamma \in[0, \pi]$.

Usually, $\mathcal{R}$ is specified by the Euler angles $\varphi, \theta, \psi$. These parameters are continuous variables, and $S O(3, \mathbb{R})$ is not the limit of a sequence of discrete subgroups. The largest discrete subgroup which covers uniformly $S O(3, \mathbb{R})$ is the icosahedron group, with 60 elements [17].

If $S O(3, \mathbb{R})$ acts on $\mathbb{R}^{3}$ by (covariant) rotations $\mathbf{e}_{k}^{\prime}=\sum_{i} \mathcal{R}_{k i}^{e} \mathbf{e}_{i}$, of the coordinate axes, the position vector $\mathbf{r}=\sum_{k} q_{k} \mathbf{e}_{k}$ of a fixed point in space remains invariant, and its coordinates are subject to the (contravariant) action $q_{k}^{\prime}=\sum_{i} \mathcal{R}_{k i}^{q} q_{i}$, where $\mathcal{R}^{q}=\left(\mathcal{R}^{e}\right)^{T}$. Using the Euler parametrization 2 these matrices take the form

$$
\mathcal{R}^{e}=\mathrm{e}^{\varphi \xi_{3}} e^{\theta \xi_{1}} e^{\psi \xi_{3}}, \mathcal{R}^{q}=\mathrm{e}^{\psi \ell_{3}} e^{\theta \ell_{1}} e^{\varphi \ell_{3}},
$$

where $\xi_{i}=-\ell_{i}, i=1,2,3$, are 3 independent, antisymmetric, $3 \times 3$ matrices, with elements $\left(\xi_{i}\right)_{j k}=-\epsilon_{i j k}\left(\epsilon_{i j k}\right.$ is the Levi-Civita symbol) and commutation relations

$$
\left[\xi_{i}, \xi_{j}\right]=\epsilon_{i j k} \xi_{k} \quad, \quad\left[\ell_{i}, \ell_{j}\right]=-\epsilon_{i j k} \ell_{k}
$$

To describe the rigid rotator, the "laboratory" frame versors $\mathbf{e}_{1}, \mathbf{e}_{2}, \mathbf{e}_{3}$ are supposed to be fixed, and a rotated frame $\mathbf{e}_{1}^{\prime}, \mathbf{e}_{2}^{\prime}, \mathbf{e}_{3}^{\prime}$ is intrinsically attached to each orientation in $\mathbb{R}^{3}$ of the rotator, such that its configuration space can be identified with $S O(3, \mathbb{R})$. If $(\varphi, \theta, \psi)$ depend on time, the derivative

$$
\dot{\mathcal{R}}^{e}=\sum_{i} \omega_{i} \xi_{i} \mathcal{R}^{e}=\mathcal{R}^{e} \sum_{i} \omega_{i}^{\prime} \xi_{i}
$$

\footnotetext{
${ }^{2}$ In quantum mechanics the second rotation is taken around the $Y$-axis, which corresponds to a change of $\varphi, \psi$ below into $\varphi+\pi / 2, \psi-\pi / 2$.
} 
defines the components of the angular velocity $\omega_{i}, \omega_{i}^{\prime},\left(\omega_{k}^{\prime}=\sum_{i} \mathcal{R}_{k i}^{q} \omega_{i}\right)$ in the laboratory, respectively in the intrinsic frame,

$$
\begin{gathered}
\omega_{1}^{\prime}=\dot{\theta} \cos \psi+\dot{\varphi} \sin \theta \sin \psi \\
\omega_{2}^{\prime}=-\dot{\theta} \sin \psi+\dot{\varphi} \sin \theta \cos \psi \\
\omega_{3}^{\prime}=\dot{\psi}+\dot{\varphi} \cos \theta .
\end{gathered}
$$

Similarly to (4) it is convenient to define the generators of the translations to the left $\left(Y_{k}\right)$ and to the right $\left(Z_{k}\right)$ by

$$
Y_{k} \mathcal{R}^{e}=\xi_{k} \mathcal{R}^{e}, \quad Z_{k} \mathcal{R}^{e}=\mathcal{R}^{e} \xi_{k}
$$

Explicitly,

$$
Y_{1}=\cos \varphi \partial_{\theta}+\frac{\sin \varphi}{\sin \theta}\left(\partial_{\psi}-\cos \theta \partial_{\varphi}\right) \quad, \quad Y_{2}=\sin \varphi \partial_{\theta}-\frac{\cos \varphi}{\sin \theta}\left(\partial_{\psi}-\cos \theta \partial_{\varphi}\right)
$$

$Y_{3}=\partial_{\varphi}$, and

$Z_{1}=\cos \psi \partial_{\theta}+\frac{\sin \psi}{\sin \theta}\left(\partial_{\varphi}-\cos \theta \partial_{\psi}\right), \quad Z_{2}=-\sin \psi \partial_{\theta}+\frac{\cos \psi}{\sin \theta}\left(\partial_{\varphi}-\cos \theta \partial_{\psi}\right)$

$Z_{3}=\partial_{\psi}$. Thus, $Z_{k}(\varphi, \theta, \psi)=-(-1)^{k} Y_{k}(\psi, \theta, \varphi)$ and

$$
\left[Y_{i}, Y_{j}\right]=-\epsilon_{i j k} Y_{k} \quad, \quad\left[Z_{i}, Z_{j}\right]=\epsilon_{i j k} Z_{k}, \quad, \quad\left[Y_{i}, Z_{j}\right]=0
$$

such that the components $x_{k}$ of a left-invariant vector field $X \in T S O(3, \mathbb{R})$, $\left(\left[X, Y_{k}\right]=0\right), X=\sum_{k} x_{k} Z_{k}$, are interpreted as "intrinsic".

The basis $Z_{k}$ of left-invariant vector fields on $T S O(3, \mathbb{R})$ can be related to a local basis $\zeta_{k}$ of one-forms on $T^{*} S O(3, \mathbb{R})$,

$$
\begin{gathered}
\zeta_{1}=\sin \theta \sin \psi d \varphi+\cos \psi d \theta \\
\zeta_{2}=\sin \theta \cos \psi d \varphi-\sin \psi d \theta \\
\zeta_{3}=\cos \theta d \varphi+d \psi,
\end{gathered}
$$

such that $\zeta_{i}\left(Z_{j}\right) \equiv\left\langle\zeta_{i}, Z_{j}\right\rangle=\delta_{i j}$. It is easy to check that

$$
d \zeta_{1}=-\zeta_{2} \wedge \zeta_{3}, d \zeta_{2}=-\zeta_{3} \wedge \zeta_{1}, d \zeta_{3}=-\zeta_{1} \wedge \zeta_{2}
$$

and $\zeta_{1} \wedge \zeta_{2} \wedge \zeta_{3}=-\sin \theta d \varphi \wedge d \theta \wedge d \psi$. Thus, if the tangent to the trajectory $\mathcal{R}^{e}(t)$ is expressed in the form $X_{\tau}=\dot{\varphi} \partial_{\varphi}+\dot{\theta} \partial_{\theta}+\dot{\psi} \partial_{\psi}$, then $\zeta_{i}\left(X_{\tau}\right)=\omega_{i}^{\prime}$ are 
the intrinsic components of the angular velocity.

The 1 -forms $\zeta_{i}$ can be used to define a symmetric 2 -form on $\operatorname{TSO}(3, \mathbb{R})$, $B=\sum_{k} I_{k} \zeta_{k} \otimes \zeta_{k}$,

$$
B(X, Y)=\sum_{k} I_{k} \zeta_{k}(X) \zeta_{k}(Y) \quad, \quad X, Y \in T S O(3, \mathbb{R})
$$

If $I_{k}>0$ are the intrinsic moments of inertia (constants), then the kinetic energy of the rigid rotator can be written in the form $T=B\left(X_{\tau}, X_{\tau}\right) / 2$. By the Legendre transform $\mathbb{L}[1$, the 2 -form $B$ provides a map $\mathbb{L}: T S O(3, \mathbb{R}) \rightarrow$ $T^{*} S O(3, \mathbb{R}), X \rightarrow \mathbb{L}_{X}, \mathbb{L}_{X}(Y) \equiv B(X, Y)$. In particular $\mathbb{L}_{X_{\tau}}=\sum_{k} \rho_{k} \zeta_{k}$, with $\rho_{k}=I_{k} \omega_{k}^{\prime}$, such that the kinetic energy $T$ also defines the classical Hamilton function $H=\sum_{k} \rho_{k}^{2} / 2 I_{k}$.

Let $\Theta=\sum_{k} \rho_{k} \zeta_{k}$ be the canonical 1-form on $M=T^{*} S O(3, \mathbb{R})$, and $\Omega=$ $-d \Theta$ the symplectic form. The dynamics of the rigid rotator can be described either as a Hamiltonian flow on $(M, \Omega)$, induced by $H$, or as geodesic motion on $S O(3, \mathbb{R})$ for the metric $B$.

\subsection{The Hamiltonian approach}

In classical mechanics the observables are represented within the set $\mathcal{F}(M)$ of the smooth real functions on the symplectic manifold $(M, \Omega)$. Let $X_{f}$ be the vector field provided by $i_{X_{f}} \Omega=d f, f \in \mathcal{F}(M)$, where $i_{X_{f}} \Omega$ denotes the inner product between $X_{f}$ and $\Omega$. The set $\mathcal{F}(M)$ becomes a Lie algebra with respect to the Poisson bracket $\{*, *\}$,

$$
\{f, g\}=\left\langle d f, X_{g}\right\rangle=\omega\left(X_{f}, X_{g}\right)=-\mathrm{L}_{X_{f}} g, \quad f, g \in \mathcal{F}(M)
$$

in which $\mathrm{L}_{X}$ denotes the Lie derivative with respect to $X$. In the case $M=$ $T^{*} S O(3, \mathbb{R}), \Omega=-d \Theta$, we get

$$
\{f, g\}=\sum_{k}\left(Z_{k} f \partial_{\rho_{k}} g-Z_{k} g \partial_{\rho_{k}} f\right)-\vec{\rho} \cdot\left(\vec{\partial}_{\rho} f \times \vec{\partial}_{\rho} g\right)
$$

where $\partial_{\rho_{k}} \equiv \partial / \partial \rho_{k}, \vec{\rho} \equiv\left(\rho_{1}, \rho_{2}, \rho_{3}\right)$ and $f, g \in \mathcal{F}(M)$ are functions $f(\mathcal{R}, \vec{\rho})$, of $\mathcal{R} \equiv \mathcal{R}^{e} \in S O(3, \mathbb{R})$ and $\vec{\rho} \in \mathbb{R}^{3}$. In particular, for the angular momentum components $\rho_{i}$ and $l_{i}=\sum_{k} \mathcal{R}_{i k} \rho_{k}$, we get $\left\{\rho_{i}, \rho_{j}\right\}=-\epsilon_{i j k} \rho_{k}$, respectively $\left\{l_{i}, l_{j}\right\}=\epsilon_{i j k} l_{k}$. A local set of canonical coordinates on $T^{*} S O(3, \mathbb{R})$, related to Wigner distribution defined in [13], is presented in Appendix 1. 
Considering the vector fields $X$ on $M$ of the form $X=\sum_{k} \omega_{k}^{\prime} Z_{k}+\dot{\rho}_{k} \partial_{\rho_{k}}$, the condition $i_{X_{H}} \Omega=d H$ reduces to the Euler equations

$$
\omega_{k}^{\prime}=\rho_{k} / I_{k} \quad, \quad \dot{\vec{\rho}}=\vec{\rho} \times \vec{\omega}^{\prime} .
$$

\subsection{The geodesic approach}

In the geodesic approach we take $B$ as a left-invariant metric on $T S O(3, \mathbb{R})$, $\mathrm{L}_{Y} B=0$. A curve $\mathcal{R}(t)$ on $S O(3, \mathbb{R})$ is a geodesic with respect to $B$ if its tangent field $X_{\tau}$ is "autoparallel", in the sense that $\nabla_{X_{\tau}} X_{\tau}=0$, where $\nabla_{X}$ is the covariant derivative with respect to $X$ induced by the metric $B$. This derivative is specified by the condition $\mathrm{L}_{X} B\left(X_{1}, X_{2}\right)=$ $B\left(\nabla_{X} X_{1}, X_{2}\right)+B\left(X_{1}, \nabla_{X} X_{2}\right)$, used to calculate the Christoffel symbols $\Gamma$. Because $\mathrm{L}_{Z_{i}} \zeta_{j}\left(Z_{k}\right)=\epsilon_{i j k}$, in the basis $\left\{Z_{k}, k=1,2,3\right\}$ of the left-invariant fields we get $\nabla_{Z_{j}} Z_{k}=\sum_{i} \Gamma_{j k}^{i} Z_{i}$ with $\Gamma_{j k}^{i}=-\left(I_{j}-I_{k}\right) \epsilon_{i j k} / I_{i}$. Taking $X_{\tau}$ of the form $X_{\tau}=\sum_{k} \omega_{k}^{\prime}(t) Z_{k}$, the condition $\nabla_{X_{\tau}} X_{\tau}=0$ yields $\dot{\omega}_{i}^{\prime}=-\sum_{j k} \Gamma_{j k}^{i} \omega_{j}^{\prime} \omega_{k}^{\prime}$, which is the same as (6). For a spherical rotator $I_{1}=I_{2}=I_{3} \equiv I, \Gamma=0$,

$$
T=\frac{I}{2}\left(\dot{\varphi}^{2}+\dot{\theta}^{2}+\dot{\psi}^{2}+2 \cos \theta \dot{\varphi} \dot{\psi}\right),
$$

and $\vec{\rho}=I \vec{\omega}^{\prime}$ is a constant. In the integrable basis $\partial_{\varphi}, \partial_{\theta}, \partial_{\psi}$, (7) corresponds to a metric tensor $\hat{g}$ having the non-vanishing components $\hat{g}_{\varphi \varphi}=\hat{g}_{\theta \theta}=\hat{g}_{\psi \psi}=1$, $\hat{g}_{\varphi \psi}=\hat{g}_{\psi \varphi}=\cos \theta\left(d v_{R}=d \varphi d \theta d \psi \sqrt{\operatorname{det} \hat{g}}\right)$ which yields the Ricci tensor $\hat{R}=\hat{g} / 2$ and the scalar curvature

$$
R=\operatorname{Tr}\left(\hat{g}^{-1} \hat{R}\right)=\frac{3}{2} .
$$

\section{The Liouville equation}

Let $\left(M_{\mu}, \Omega_{\mu}\right)$ be the classical phase-space of an elementary rotator $\mu$ (e.g. molecule), and $\left(M_{\Gamma}, \Omega_{\Gamma}\right)$ the phase-space of the ensemble (gas) consisting of $N$ identical elementary rotators [18],

$$
M_{\Gamma}=M_{1} \times M_{2} \times \ldots M_{N} \quad, \quad \Omega_{\Gamma}=\sum_{\mu=1}^{N} \Omega_{\mu} .
$$


For a statistical description of the ensemble we presume that on each manifold $M_{\mu}$ can be defined a partition in $K$ infinitesimal cells, $\left\{b_{j} ; j=1, K\right\}$, of volume $\delta v^{j}=\int_{b_{j}} d v_{R} d^{3} \rho$,

$$
d v_{R} \equiv \sin \theta d \varphi d \theta d \psi \quad, \quad d^{3} \rho \equiv d \rho_{1} d \rho_{2} d \rho_{3} .
$$

This partition induces a partition of $M_{\Gamma}$ in $n_{B}=K^{N}$ cells $B_{j}$ of volume $\delta V_{\Gamma}^{j}, j=1, n_{B}$. Denoting by $w_{j}$ the probability of finding the representative point $m \in M_{\Gamma}$, for the state of the ensemble 3 at the time $t$, localized in $B_{j}$, the ratio $\mathrm{F}_{j}=w_{j} / \delta V_{\Gamma}^{j}$ defines the distribution function $\mathrm{F}$ of the probability density. This is symmetric at the permutation of the rotator indices and normalized by

$$
\int_{M_{\Gamma}} d V_{\Gamma} \mathrm{F}=1, \quad d V_{\Gamma}=\Pi_{\mu=1}^{N}\left(d v_{R} d^{3} \rho\right)_{\mu}
$$

It is important to remark that a definition of $F$ with respect to the partition $\left\{B_{j} ; j=1, n_{B}\right\}$ does not ensure $\mathrm{F} \in \mathcal{F}\left(M_{\Gamma}\right)$. A more suitable description would require a covering of $M_{\Gamma}$, defined as an indexed system of open sets $\left\{U_{i}, i \in I\right\}$, such that $\cup_{i} U_{i}=M_{\Gamma}$, and a system of $q$-cochains [19], $q=0,1$, associating to each set of $q+1$ indices $i_{0}, \ldots, i_{q}$ from $I$ a function $\mathrm{F}_{q}\left(i_{0}, \ldots, i_{q}\right) \in \mathbb{R}$ on $U_{i_{0}} \cap U_{i_{1}} \ldots \cap U_{i_{q}}$.

Because $d v_{R} d^{3} \rho=\left|\Omega^{3}\right|$, the volume element $d V_{\Gamma}$ is invariant to the Hamiltonian flow on $M_{\Gamma}$, and $\mathrm{F}$ evolves according to the continuity (Liouville) equation

$$
\partial_{t} \mathrm{~F}=\left\{H_{\Gamma}, \mathrm{F}\right\}_{\Gamma},
$$

where $\{,\}_{\Gamma}$ is the Poisson bracket on $M_{\Gamma}$ and $H_{\Gamma}$ is the Hamiltonian of the ensemble. The mean value of an observable $A \in \mathcal{F}\left(M_{\Gamma}\right)$ is

$$
<A>_{\mathrm{F}}=\int_{M_{\Gamma}} d V_{\Gamma} \mathrm{F} A,
$$

and

$$
\frac{d<A>_{\mathrm{F}}}{d t}=<\left\{A, H_{\Gamma}\right\}_{\Gamma}>_{\mathrm{F}}
$$

\footnotetext{
${ }^{3}$ The permutations of the rotators yield $N$ ! possible representative points for the same physical state of the ensemble.
} 
For an ensemble of non-interacting rotators a particular solution of (11) is $\mathrm{F}(m, t)=\Pi_{\mu=1}^{N} \mathrm{f}\left(\mathcal{R}_{\mu}, \vec{\rho}_{\mu}, t\right)$, where $\mathrm{f} \in \mathcal{F}\left(M_{\mu}\right)$ is a solution of the Liouville equation on $T^{*} S O(3, \mathbb{R}), \partial_{t} \mathrm{f}=\{H, \mathrm{f}\}$, with $H=\sum_{k} \rho_{k}^{2} / 2 I_{k}$, namely

$$
\partial_{t} \mathrm{f}+\sum_{k} \frac{\rho_{k}}{I_{k}} Z_{k} \mathrm{f}+\sum_{i j k} \epsilon_{i j k} \rho_{i} \frac{\rho_{j}}{I_{j}} \partial_{\rho_{k}} \mathrm{f}=0 .
$$

Let $\tilde{f}$ be the Fourier transform of $f$ with respect to the intrinsic angular momentum,

$$
\tilde{\mathbf{f}}(\mathcal{R}, \mathbf{r}, t) \equiv \int d^{3} \rho \mathrm{e}^{\mathrm{ir} \cdot \vec{\rho}} \mathrm{f}(\mathcal{R}, \vec{\rho}, t)
$$

Thus, if $\mathrm{f}(\mathcal{R}, \vec{\rho}, t)$ is a solution of (13) , then $\tilde{\mathrm{f}}(\mathcal{R}, \mathbf{r}, t)$ will satisfy

$$
\partial_{t} \tilde{\mathrm{f}}-\mathrm{i} \sum_{i} \frac{1}{I_{i}} Z_{i} \partial_{r_{i}} \tilde{\mathrm{f}}+\mathrm{i} \sum_{i j k} \epsilon_{i j k} \frac{r_{i}}{I_{k}} \partial_{r_{j}} \partial_{r_{k}} \tilde{\mathrm{f}}=0
$$

Particular solutions of this equation are related to local Lagrangian submanifolds $\Lambda \subset T^{*} S O(3, \mathbb{R})$, such that $\left.\Theta\right|_{\Lambda}=d S$, where $S(\mathcal{R}, t)$ is the generating function of the Hamilton-Jacobi theory. These solutions are of the form

$$
\tilde{\mathrm{f}}_{0}(\mathcal{R}, \mathbf{r}, t)=\mathrm{n}(\mathcal{R}, t) \mathrm{e}^{\mathrm{i} \mathbf{r} \cdot \mathbf{Z} S}
$$

where the density $\mathrm{n} \geq 0$ and $S$ are real functions on $S O(3, \mathbb{R})$ which satisfy the continuity and, respectively, the Hamilton-Jacobi equations

$$
\partial_{t} \mathrm{n}+\sum_{k} Z_{k}\left(\mathrm{n} \frac{Z_{k} S}{I_{k}}\right)=0 \quad, \quad Z_{k}\left[\partial_{t} S+\sum_{k} \frac{\left(Z_{k} S\right)^{2}}{2 I_{k}}\right]=0
$$

The inverse of (14),

$$
\mathrm{f}(\mathcal{R}, \vec{\rho}, t)=\frac{1}{(2 \pi)^{3}} \int d^{3} \mathrm{r} \mathrm{e}^{-\mathrm{ir} \cdot \vec{\rho}} \tilde{\mathrm{f}}(\mathcal{R}, \mathbf{r}, t)
$$

takes in this case the form of the classical "action distributions",

$$
\mathrm{f}_{0}(\mathcal{R}, \vec{\rho}, t)=\mathrm{n} \delta(\vec{\rho}-\mathbf{Z} S)
$$

These are coherent solutions of (13) in the sense that during time evolution remain the same functionals of $\mathrm{n}$ and $S$.

The quantum (Wigner-type) distributions are related to a peculiar form 
of $\tilde{\mathbf{f}}(\mathcal{R}, \mathbf{r}, t)$, in which the variable $\mathbf{r}$ (Fourier dual to $\vec{\rho}$ ) enters as a parameter for translations on the configuration space. To obtain this functional we note that in (16) $\mathbf{Z}$ is the generator of the translations to the right, and therefore

$$
\mathbf{r} \cdot \mathbf{Z} S(\mathcal{R})=\lim _{\sigma \rightarrow 0} \frac{1}{\sigma}\left[S\left(\mathcal{R} \mathrm{e}^{\sigma \mathbf{r} \cdot \vec{\xi} / 2}\right)-S\left(\mathcal{R} \mathrm{e}^{-\sigma \mathbf{r} \cdot \vec{\xi} / 2}\right)\right]
$$

where $\sigma$ is a real parameter, $\sigma \mathbf{r} \cdot \vec{\xi}$ is an element of the Lie algebra $\mathfrak{s} o(3, \mathbb{R})$, and $\gamma=\sigma|\mathbf{r}|$ has the significance of a rotation angle. On the Cartan subalgebra of any semisimple Lie algebra we can introduce a lattice structure, dual to the lattice of the weights, while discrete rotation angles are associated with a complete orthonormal set of angle states [20, 21]. Moreover, the commutation relations $\left[Z_{i}, Z_{j}\right]=\epsilon_{i j k} Z_{k}$ are due to the nonlinearity of the operator $\mathbf{Z}$, which is effective only outside a certain "infinitesimal domain" around identity of the angular coordinates. Thus, near $\mathbf{r}=0$ the derivative (20) may retain the nonlocal, finite differences expression, in which $\sigma$ is a small, but finite constant. Considering $\sigma=\hbar$, (16) suggests the "quantum" form

$$
\tilde{\mathrm{f}}_{\Psi}(\mathcal{R}, \mathbf{r}, t)=\Psi\left(\mathcal{R} \mathrm{e}^{\hbar \mathbf{r} \cdot \vec{\xi} / 2}\right) \Psi^{*}\left(\mathcal{R} \mathrm{e}^{-\hbar \mathbf{r} \cdot \vec{\xi} / 2}\right)
$$

where $\Psi=\sqrt{\mathrm{n}} \mathrm{e}^{\mathrm{i} S / \hbar}$ is the complex wave function. This expression, in principle, will provide by (18) a phase-space distribution $\mathrm{f}_{\Psi}$, but due to the infinite integration domain over $\mathbf{r}$, such a functional will not have the properties of the Wigner quasiprobability distributions. Therefore, at $\rho / \hbar$ large, we may consider instead the functional

$$
\mathrm{f}_{W}(\mathcal{R}, \vec{\rho}, t)=\frac{1}{(2 \pi)^{3}} \int_{r \leq \pi / \hbar} d^{3} \mathrm{r} j_{0}^{2}\left(\frac{\hbar r}{2}\right) \mathrm{e}^{-\mathrm{i} \mathbf{r} \cdot \vec{\rho}} \tilde{\mathbf{f}}_{\Psi}(\mathcal{R}, \mathbf{r}, t)
$$

where $r=|\mathbf{r}|$ and $j_{0}(x)=x^{-1} \sin x$. Using the new variable $\vec{\gamma}=\hbar \mathbf{r}$, (21) can also be written in the form

$$
\mathrm{f}_{W}(\mathcal{R}, \vec{\rho})=\frac{1}{(2 \pi \hbar)^{3}} \int_{\gamma \leq \pi} d^{3} \gamma j_{0}^{2}\left(\frac{\gamma}{2}\right) \mathrm{e}^{-\mathrm{i} \vec{\gamma} \cdot \vec{\rho} / \hbar} \Psi\left(\mathcal{R} \mathrm{e}^{\vec{\gamma} \cdot \vec{\xi} / 2}\right) \Psi^{*}\left(\mathcal{R} \mathrm{e}^{-\vec{\gamma} \cdot \vec{\xi} / 2}\right)
$$

The vector $\vec{\gamma}=\left(\gamma_{1}, \gamma_{2}, \gamma_{3}\right)=\gamma \mathbf{g}, \mathbf{g}=(\cos \alpha \sin \beta, \sin \alpha \sin \beta, \cos \beta)$, yields the parametrization of the rotation matrices by the exponential map $\mathcal{R}=$ $\exp \left(\gamma_{1} \xi_{1}+\gamma_{2} \xi_{2}+\gamma_{3} \xi_{3}\right)$, and is related to the Euler angles by

$$
\tan \varphi=-\frac{\cos \beta(1-\cos \gamma) \cos \alpha+\sin \gamma \sin \alpha}{\cos \beta(1-\cos \gamma) \sin \alpha-\sin \gamma \cos \alpha}
$$

\footnotetext{
${ }^{4} \gamma$ provides the character $\chi_{1}(\mathcal{R})=\operatorname{Tr}(\mathcal{R})=2 \cos \gamma+1$.
} 


$$
\tan \psi=\frac{\cos \beta(1-\cos \gamma) \cos \alpha-\sin \gamma \sin \alpha}{\cos \beta(1-\cos \gamma) \sin \alpha+\sin \gamma \cos \alpha}
$$

$\cos \theta=1-2 \sin ^{2} \beta \sin ^{2}(\gamma / 2)$, and

$$
d \varphi=d \alpha-a\left(d \beta-\frac{\cot \beta}{\sin \gamma} d \gamma\right), \quad d \psi=-d \alpha-a\left(d \beta-\frac{\cot \beta}{\sin \gamma} d \gamma\right)
$$

where $a=\sin \beta \sin \gamma /\left[2-2 \sin ^{2} \beta \sin ^{2}(\gamma / 2)\right]$. The factor $j_{0}^{2}(\gamma / 2), \gamma=|\vec{\gamma}|$ (found also in [17]-14.7), was introduced such that $j_{0}^{2}(\gamma / 2) d \gamma_{1} d \gamma_{2} d \gamma_{3}$ becomes in the Euler parametrization the volume element $d v_{R}=\sin \theta d \varphi d \theta d \psi$. In this case, if $\mathrm{f}_{W 1}$ and $\mathrm{f}_{W 2}$ are given by $\Psi_{1}$, respectively $\Psi_{2}$, then

$$
<\mathrm{f}_{W 1}>_{\mathrm{f}_{W 2}}=\left|\left\langle\Psi_{1} \mid \Psi_{2}\right\rangle\right|^{2} /(2 \pi \hbar)^{3} \text {. }
$$

The mean values of the intrinsic angular momentum components,

$$
\begin{gathered}
<\rho_{k}>_{\mathrm{f}_{W}}=\int d^{3} \rho d v_{R} \rho_{k} \mathrm{f}_{W}= \\
\frac{1}{(2 \pi \hbar)^{3}} \int d^{3} \rho d v_{R} \int_{\gamma \leq \pi} d^{3} \gamma j_{0}^{2}\left(\frac{\gamma}{2}\right)\left(\mathrm{i} \hbar \partial_{\gamma_{k}} \mathrm{e}^{-\mathrm{i} \vec{\gamma} \cdot \vec{\rho} / \hbar}\right) \Psi\left(\mathcal{R} \mathrm{e}^{\vec{\gamma} \cdot \vec{\xi} / 2}\right) \Psi^{*}\left(\mathcal{R} \mathrm{e}^{-\vec{\gamma} \cdot \vec{\xi} / 2}\right)
\end{gathered}
$$

can be estimated considering that the integral over $d^{3} \rho$ yields a delta function $\delta(\vec{\gamma})$, the first derivative of $j_{0}^{2}(\gamma / 2)$ vanishes at $\gamma=0$, and

$$
\left.\partial_{\gamma_{i}} \Psi\left(\mathcal{R} \mathrm{e}^{\vec{\gamma} \cdot \vec{\xi} / 2}\right)\right|_{\gamma=0}=\frac{1}{2} Z_{i} \Psi(\mathcal{R})
$$

such that

$$
<\rho_{k}>_{\mathrm{f}_{W}}=\frac{1}{2} \int d v_{R}\left[\left(\hat{L}_{k}^{\prime} \Psi\right) \Psi^{*}+\Psi\left(\hat{L}_{k}^{\prime} \Psi\right)^{*}\right]
$$

where $\hat{L}_{k}^{\prime}=-\mathrm{i} \hbar Z_{k}$ is the intrinsic angular momentum operator. This operator is Hermitian with respect to the volume element $d v_{R}$, and therefore $<\rho_{k}>_{\mathrm{f}_{W}}=\left\langle\Psi\left|\hat{L}_{k}^{\prime}\right| \Psi\right\rangle$.

The calculation of $\left\langle\rho_{k}^{2}>_{f_{W}}\right.$ involves second derivatives with respect to $\gamma_{k}$ and proceeds similarly, excepting for the second derivative $\partial_{\gamma_{k}}^{2} j_{0}^{2}(\gamma / 2)$ at $\gamma=0$, which is $-1 / 6$. Thus, the operator associated to $\rho_{k}^{2}$ by $\mathrm{f}_{W}$ is $\left(\hat{L}_{k}^{\prime}\right)^{2}+\hbar^{2} / 6$, and the Hamiltonian operator

$$
\hat{H}=\sum_{k}\left(\frac{\hat{L}_{k}^{\prime}}{2 I_{k}}+\frac{\hbar^{2}}{12 I_{k}}\right)
$$


contains beside the usual part a zero-point energy term $\epsilon_{0}=\sum_{k} \hbar^{2} / 12 I_{k}$. For a spherical rotator $\epsilon_{0}=\hbar^{2} / 4 I=\hbar^{2} R / 6 I$, where $R$ is the scalar curvature 5 (8), in agreement with [9].

Because $j_{0}(0)=1$, from (21) we get the limit $\lim _{\hbar \rightarrow 0} f_{W}=f_{0}$, which is a coherent distribution. It can also be shown that a necessary condition for $f_{W}$ to be a solution of the Liouville equation is that $\mathrm{i} \hbar \partial_{t} \Psi=\hat{H} \Psi$ (Appendix 2).

A more general expression of $\mathrm{f}_{W}$ can be obtained by writing the factor $\mathrm{e}^{-\mathrm{i} \vec{\gamma} \cdot \vec{\rho} / \hbar}$ from (22) in terms of the 1 -form $\Theta_{\rho}=\sum_{k} \rho_{k} \zeta_{k}$, where $\rho_{k}$ are constants. Because $\mathbf{g} \cdot \mathbf{Z}=\partial_{\gamma}$, we get $\vec{\gamma} \cdot \vec{\rho}=\gamma\left\langle\Theta_{\rho}, \partial_{\gamma}\right\rangle=\hbar \Phi_{\rho}\left(\mathcal{R}_{\gamma}, \mathcal{L}_{\gamma}\right)$, where the phase

$$
\Phi_{\rho}\left(\mathcal{R}_{\gamma}, \mathcal{L}_{\gamma}\right)=\left.\frac{1}{\hbar} \int_{\mathcal{R}_{\gamma}^{-1 / 2}}^{\mathcal{R}_{\gamma}^{1 / 2}} \Theta_{\rho}\right|_{\mathcal{L}_{\gamma}}, \quad \mathcal{R}_{\gamma}=\mathrm{e}^{\vec{\gamma} \cdot \vec{\xi}}
$$

is the integral of $\Theta_{\rho} / \hbar$ along the line $\mathcal{L}_{\gamma}=\left\{\mathrm{e}^{\mathrm{tg} \cdot \vec{\xi}}, t \in[-\gamma / 2, \gamma / 2]\right\}$ between $\mathcal{R}_{\gamma}^{-1 / 2}$ and $\mathcal{R}_{\gamma}^{1 / 2}$. An intrinsic expression of $\mathrm{f}_{W}$, independent of coordinates, should be therefore of the form

$$
\mathrm{f}_{W}(\mathcal{R}, \vec{\rho},[\mathcal{C}])=\frac{1}{(2 \pi \hbar)^{3}} \int_{S O 3} d v_{\tilde{R}} \mathrm{e}^{-\mathrm{i} \Phi_{\rho}(\tilde{\mathcal{R}}, \mathcal{C})} \Psi\left(\mathcal{R} \tilde{\mathcal{R}}^{1 / 2}\right) \Psi^{*}\left(\mathcal{R} \tilde{\mathcal{R}}^{-1 / 2}\right)
$$

where $\mathcal{C} \in[\mathcal{C}]$ is a curve of a suitable type.

It is important to remark that $X_{\rho}=\vec{\rho} \cdot \mathbf{Z}$ is a characteristic vector of $\omega_{\rho}=-d \Theta_{\rho},\left(i_{X_{\rho}} \omega_{\rho}=0\right)$, such that if $G_{\rho} \subset S O(3, \mathbb{R})$ is the stability subgroup of $\vec{\rho}$, then $M_{\rho}=S O(3, \mathbb{R}) / G_{\rho}$ has a symplectic structure given by the 2 -form $\omega_{o}, \pi^{*} \omega_{o}=\omega_{\rho}$, where $\pi$ is the projection $\pi: S O(3, \mathbb{R}) \rightarrow M_{\rho}$ along the orbits of $X_{\rho}$.

\section{Concluding remarks}

The Wigner transform defines quasiprobability distributions on the phasespace $M=T^{*} \mathbb{R}^{3}$ which evolve as coherent solutions of the classical Liouville equation for particles subject to uniform or elastic force fields [14]. It also relates classical and quantum expectation values for many observables and provides a basis for the statistical interpretation of the scalar product between quantum wave functions. However, to find the geometrical structure

\footnotetext{
${ }^{5}$ In the basis $\partial_{\alpha}, \partial_{\beta}, \partial_{\gamma}$ the metric tensor $\hat{g}$ is diagonal, with $\hat{g}_{\alpha \alpha}=\hat{g}_{\beta \beta} \sin ^{2} \beta, \hat{g}_{\beta \beta}=$ $4 \sin ^{2}(\gamma / 2), \hat{g}_{\gamma \gamma}=1$.
} 
underlying these properties, it is necessary to go beyond $T^{*} \mathbb{R}^{3}$.

The case $M=T^{*} S O(3, \mathbb{R})$ corresponds to the rigid rotator, and the proposed extension is $f_{W}$ of (22). This functional reduces to the "action distribution" $\mathrm{f}_{0}(19)$ in the limit $\hbar \rightarrow 0$ and ensures the usual quantization of the intrinsic angular momentum. Though, the classical expectation value of the intrinsic Hamiltonian contains beside the usual "quantum part", a zeropoint energy term. This term is positive definite and reflects the compact, rather than the non-abelian structure of $S O(3, \mathbb{R})$.

Formally, a local distribution function on $T^{*} S O(3, \mathbb{R})$ can also be defined as reduced Wigner transform on $T^{*} \mathbb{R}^{4}$, (Appendix 1). Compared to this, the intrinsic approach has the advantage of providing more insight into the fundamental aspects of the transition between classical and quantum distributions, by taking into account the specific geometry of the problem.

Appendix 1: the $T^{*} \mathbb{R}^{4} \rightarrow T^{*} S O(3, \mathbb{R})$ reduction

The covering group of $S O(3, \mathbb{R})$ is $S U(2)$, which is homeomorphic to the sphere $S^{3} \subset \mathbb{R}^{4}[22]$. Thus, a quasiprobability density on $T^{*} S O(3, \mathbb{R})$ can be obtained by reduction from the standard Wigner transform of an extended wave function $\Psi_{e}(X)$ on $\mathbb{R}^{4}$,

$$
\mathrm{f}_{e}(X, P)=\frac{1}{(2 \pi)^{4}} \int d^{4} K \mathrm{e}^{-\mathrm{i} K \cdot P} \Psi_{e}\left(X+\frac{\hbar}{2} K\right) \Psi_{e}^{*}\left(X-\frac{\hbar}{2} K\right)
$$

where $X, P, K$ are 4 -vectors of the form $X=\left(x_{1}, x_{2}, x_{3}, x_{4}\right)$, specified by the Cartesian components $x_{i}$. Let $(r, \theta, \nu, \eta)$ be the spherical coordinates on $\mathbb{R}^{4}$, $\theta \in[0, \pi], \nu, \eta \in[0,2 \pi]$, such that

$$
\begin{aligned}
& x_{1}=r \cos \frac{\theta}{2} \cos \nu, x_{2}=r \cos \frac{\theta}{2} \sin \nu \\
& x_{3}=r \sin \frac{\theta}{2} \cos \eta, x_{4}=r \sin \frac{\theta}{2} \sin \eta .
\end{aligned}
$$

Thus, $\sum_{k} x_{k}^{2}=r^{2}$, and for $r=1$ we get

$$
\hat{a}_{X}=\left[\begin{array}{cc}
x_{1}+\mathrm{i} x_{2} & x_{3}-\mathrm{i} x_{4} \\
-x_{3}-\mathrm{i} x_{4} & x_{1}-\mathrm{i} x_{2}
\end{array}\right] \in S U(2) .
$$

The volume element $d^{4} X \equiv d x_{1} d x_{2} d x_{3} d x_{4}=r^{3} \sin \theta d \theta d \nu d \eta d r / 4$ yields for the ball of radius $R$ the volume $V_{B 4}=\pi^{2} R^{4} / 2$. Thus, the "area" of the unit 
sphere $S^{3}$, which can be taken also as the volume of $S U(2)$, is $V_{S U 2}=2 \pi^{2}$.

In spherical coordinates, the canonical symplectic form on $T^{*} \mathbb{R}^{4}, \omega_{e}=$ $\sum_{k} d x_{k} \wedge d p_{k}, p_{k}=m \dot{x}_{k}$, takes the form 6

$$
\begin{gathered}
\omega_{e}=d r \wedge d p_{r}+d \theta \wedge d p_{\theta}+d \nu \wedge d p_{\nu}+d \eta \wedge d p_{\eta}, \\
p_{r}=m \dot{r} \quad, \quad p_{\theta}=m \frac{r^{2}}{4} \dot{\theta} \quad, \quad p_{\nu}=m r^{2} \cos ^{2} \frac{\theta}{2} \dot{\nu}, \quad p_{\eta}=m r^{2} \sin ^{2} \frac{\theta}{2} \dot{\eta},
\end{gathered}
$$

providing the volume element on $T^{*} \mathbb{R}^{4},\left|\omega_{e}^{4}\right|=d r d \theta d \nu d \eta d p_{r} d p_{\theta} d p_{\nu} d p_{\eta}$. Because $\int\left|\omega_{e}^{4}\right| f_{e}(X, P)=1$, the reduced Wigner distribution on $T^{*} S U(2)$ is defined by

$$
\mathrm{f}_{S U 2}\left(\theta, \nu, \eta, p_{\theta}, p_{\nu}, p_{\eta}\right)=\int_{0}^{\infty} d r \int_{-\infty}^{\infty} d p_{r} \mathrm{f}_{e}(X, P)
$$

The projection $S U(2) \rightarrow S O(3, \mathbb{R})$ consists in the identification of the $S^{3}$ poles $X=(1,0,0,0)$ and $(-1,0,0,0)$. Thus, $\mathrm{f}_{S U 2}$ provides further a distribution $\mathrm{f}_{S O 3}$ on $T^{*} S O(3, \mathbb{R})$, expressed in terms of the Euler angles $\theta, \varphi, \psi$ and the related momenta $p_{\theta}, p_{\varphi}, p_{\psi}$, if it remains well defined after the change of coordinates

$$
\begin{gathered}
\nu=\frac{\varphi+\psi}{2}, \quad \eta=\frac{\varphi-\psi}{2}, \\
p_{\nu}=p_{\varphi}+p_{\psi} \quad, \quad p_{\eta}=p_{\varphi}-p_{\psi} .
\end{gathered}
$$

The volume of the phase-space element is invariant at this projection, but we should note that the volume of the configuration space decreases by a factor of 2 .

\section{Appendix 2: the coherence of the quantum distributions}

Theorem. Let $\tilde{f}_{W}$ be the Fourier transform in momentum of the quantum quasiprobability distribution $\mathrm{f}_{W}$ on $T^{*} S O(3, \mathbb{R})$,

$$
\tilde{\mathbf{f}}_{W}(\mathcal{R}, \mathbf{r}, t) \equiv \int d^{3} \rho \mathrm{e}^{\mathrm{i} \mathbf{r} \cdot \vec{\rho}} \mathrm{f}_{W}(\mathcal{R}, \vec{\rho}, t)
$$

Then, in the limit $\mathbf{r} \rightarrow 0$ :

i) $\tilde{\mathrm{f}}_{W}(\mathcal{R}, \mathbf{r}) \sim\left(\hat{U}_{\gamma} \Psi\right)_{(\mathcal{R})}\left(\hat{U}_{-\gamma} \Psi^{*}\right)_{(\mathcal{R})}, \hat{U}_{ \pm \gamma}=\mathrm{e}^{ \pm \vec{\gamma} \cdot \mathbf{Z} / 2}, \vec{\gamma}=\hbar \mathbf{r}$, and

ii) a necessary condition for $f_{W}$ to satisfy the Liouville equation (13) $\partial_{t} f_{W}=$

\footnotetext{
${ }^{6}$ For a particle of mass $m$ on $T^{*} \mathbb{R}^{3}, \omega=\sum_{k} d x_{k} \wedge d p_{k}=d r \wedge d p_{r}+d \theta \wedge d p_{\theta}+d \varphi \wedge d p_{\varphi}$, with $p_{r}=m \dot{r}, p_{\theta}=m r^{2} \dot{\theta}, p_{\varphi}=m r^{2} \sin ^{2} \theta \dot{\varphi}$.
} 
$\left\{H, \mathrm{f}_{W}\right\}$ is that $\Psi$ evolves according to the time-dependent Schrödinger equation $\mathrm{i} \hbar \partial_{t} \Psi=\hat{H} \Psi$.

Proof. The first assertion was discussed in Sect. 3, at the definition of $f_{W}$. Thus, by the transform (24) of (21) we get

$$
\tilde{\mathrm{f}}_{W}(\mathcal{R}, \mathbf{r})=j_{0}^{2}(\gamma / 2) \Psi\left(\mathcal{R} \mathrm{e}^{\vec{\gamma} \cdot \vec{\xi} / 2}\right) \Psi^{*}\left(\mathcal{R} \mathrm{e}^{-\vec{\gamma} \cdot \vec{\xi} / 2}\right), \quad \vec{\gamma}=\hbar \mathbf{r}
$$

in which $j_{0}^{2}(\gamma / 2)$ decreases slowly from 1 to 0.4 when $\gamma \in[0, \pi]$.

For ii) we first obtain from (24)

$$
\left(\partial_{t}-\mathrm{i} \hbar \hat{\mathbf{a}} \cdot \hat{\mathbf{b}}\right) \tilde{\mathbf{f}}_{W}=\int d^{3} \rho \mathrm{e}^{\mathrm{i} \vec{\gamma} \cdot \vec{\rho} / \hbar}\left(\partial_{t} \mathrm{f}_{W}-\left\{H, \mathrm{f}_{W}\right\}\right)
$$

where $\hat{\mathbf{a}}, \hat{\mathbf{b}}$ are the operators $\hat{a}_{i}=I_{i}^{-1} \partial_{\gamma_{i}}, \hat{b}_{i}=Z_{i}-\sum_{j k} \epsilon_{i j k} \gamma_{j} \partial_{\gamma_{k}}$. Thus, if $\mathrm{f}_{W}$ is an exact (coherent) solution of (13) then $\left(\partial_{t}-\mathbf{i} \hbar \hat{\mathbf{a}} \cdot \hat{\mathbf{b}}\right) \tilde{\mathbf{f}}_{W}=0$. To calculate $\hat{b}_{i} \tilde{f}_{W}$ we note that $\mathbf{Z}$ acts only on the parameters of $\mathcal{R}$, such that

$$
\hat{\mathbf{b}} \tilde{\mathbf{f}}_{W}=j_{0}^{2}(\gamma / 2)\left[\left(\hat{\mathbf{b}} \hat{U}_{\gamma} \Psi\right)\left(\hat{U}_{-\gamma} \Psi^{*}\right)+\left(\hat{U}_{\gamma} \Psi\right)\left(\hat{\mathbf{b}} \hat{U}_{-\gamma} \Psi^{*}\right)\right]
$$

Let $\mathbf{Y}^{\gamma}$ and $\mathbf{Z}^{\gamma}$ be the generators of the left and right translations for the element $\mathcal{R}_{\gamma}=\exp (\vec{\gamma} \cdot \vec{\xi})$, with $\vec{\gamma}=\gamma \mathbf{g}$. Explicitly

$Y_{i}^{\gamma}=\partial_{\gamma_{i}}+\frac{f-1}{\gamma} \nabla_{i}^{A}+\frac{1}{2}\left(\mathbf{g} \times \nabla^{A}\right)_{i}, \quad Z_{i}^{\gamma}=\partial_{\gamma_{i}}+\frac{f-1}{\gamma} \nabla_{i}^{A}-\frac{1}{2}\left(\mathbf{g} \times \nabla^{A}\right)_{i}$,

where $f(\gamma)=(\gamma / 2) \cot (\gamma / 2)$ and $\nabla^{A}$ is the angular part of $\partial_{\gamma_{i}}=\mathbf{g}_{i} \partial_{\gamma}+\nabla_{i}^{A} / \gamma$ (similar expressions have been derived in [23]). Thus, $\hat{\lambda}=\mathbf{g} \times \nabla^{A}=\mathbf{Y}^{\gamma}-\mathbf{Z}^{\gamma}$ is independent of $\gamma$ and acts only on $\mathbf{g}$ (the angular part of $\vec{\gamma}$, configuration space for the simple rotator), such that $\hat{\lambda}_{i} \hat{U}_{ \pm \gamma}=Z_{i} \hat{U}_{ \pm \gamma}-\hat{U}_{ \pm \gamma} Z_{i}$. However, $\hat{b}_{i}=Z_{i}-\hat{\lambda}_{i}, \hat{b}_{i} \hat{U}_{ \pm \gamma}=\left(Z_{i}-\hat{\lambda}_{i}\right) \hat{U}_{ \pm \gamma}=\hat{U}_{ \pm \gamma} Z_{i}$, and (25) becomes

$$
\hat{b}_{i} \tilde{\mathrm{f}}_{W}=j_{0}^{2}\left(\frac{\gamma}{2}\right)\left[\left(\hat{U}_{\gamma} Z_{i} \Psi\right)\left(\hat{U}_{-\gamma} \Psi^{*}\right)+\left(\hat{U}_{\gamma} \Psi\right)\left(\hat{U}_{-\gamma} Z_{i} \Psi^{*}\right)\right]
$$

The action of $\hat{a}_{i}$ on this function takes a simple form only if $\vec{\gamma}=\hbar \mathbf{r}$ is small or along the $i$-axis, when $\hat{U}_{\gamma}=\mathrm{e}^{\gamma_{i} Z_{i} / 2}$ and $\partial_{\gamma_{i}} \hat{U}_{\gamma}=\left(Z_{i} / 2\right) \hat{U}_{\gamma}=\hat{U}_{\gamma} Z_{i} / 2$. Thus, presuming that $\gamma$ is small and $\hat{a}_{i} \hat{U}_{ \pm \gamma} \sim \pm \hat{U}_{ \pm \gamma} Z_{i} / 2 I_{i}$, we get

$$
-\hbar^{2} \hat{\mathbf{a}} \cdot \hat{\mathbf{b}} \tilde{\mathbf{f}}_{W} \sim\left[\left(\hat{U}_{\gamma} \hat{H} \Psi\right)\left(\hat{U}_{-\gamma} \Psi^{*}\right)-\left(\hat{U}_{\gamma} \Psi\right)\left(\hat{U}_{-\gamma} \hat{H} \Psi^{*}\right)\right]
$$


with $\hat{H}=-\hbar^{2} \sum_{i} Z_{i}^{2} / 2 I_{i}$ (up to a constant). Introducing $\hat{\Lambda}=\partial_{t}+\mathrm{i} \hat{H} / \hbar$,

$$
\left(\partial_{t}-\mathrm{i} \hbar \hat{\mathbf{a}} \cdot \hat{\mathbf{b}}\right) \tilde{\mathbf{f}}_{W} \sim\left[\left(\hat{U}_{\gamma} \hat{\Lambda} \Psi\right)\left(\hat{U}_{-\gamma} \Psi^{*}\right)+\left(\hat{U}_{\gamma} \Psi\right)\left(\hat{U}_{-\gamma} \hat{\Lambda}^{*} \Psi^{*}\right)\right]
$$

such that if $\mathrm{f}_{W}$ is a solution of the classical Liouville equation then $\hat{\Lambda} \Psi=0$, namely $\Psi$ is a solution of the time-dependent Schrödinger equation $\mathrm{i} \hbar \partial_{t} \Psi=$ $\hat{H} \Psi$. In general, we may expect that the coherence (functional form) of $\mathrm{f}_{W}$ is maintained as long as $\tilde{f}_{W}(\mathcal{R}, \mathbf{r})$ has significant, non-vanishing values only for $\hbar|\mathbf{r}| \ll \pi$.

\section{References}

[1] J. E. Marsden, Lectures on Mechanics, Cambridge (1992).

[2] A. Faessler, D. T. Khoa, M. Grigorescu and R. Nojarov, Low-lying magnetic dipole excitations in actinide nuclei, Phys. Rev. Lett. 652978 (1990).

[3] M. Grigorescu, D. Rompf and W. Scheid, Dynamical effects of deformation in the coupled two-rotor system, Phys. Rev. C 571218 (1998).

[4] M. Grigorescu, Low-lying isovector monopole resonances, J. Phys. G: Nucl. Part. Phys. 16417 (1990).

[5] M. Grigorescu, Structure effects of the two-protons two-neutrons correlations, Rev. Roum. Phys. 37107 (1992).

[6] L. Landau and E. Lifchitz, Physique Statistique, Éditions Mir, Moscou (1967).

[7] A. Einstein and O. Stern, Einige Argumente für die Annahme einer molekular Agitation beim absoluten Nullpunkt, Ann. Phys. 40 551-560 (1913).

[8] J. Śniatycki, Geometric Quantization and Quantum Mechanics, Springer, New York (1980), p. 21.

[9] N. E. Hurt, Geometric Quantization in Action: Applications of Harmonic Analysis in Quantum Statistical Mechanics and Quantum Field Theory, Reidel, Dordrecht (1980), p. 69. 
[10] C. T. Prieto, Quantization and spectral geometry of a rigid body in a magnetic monopole field, Diff. Geom. Appl. 14 157-179 (2001).

[11] C. P. Malta, T. S. Marshall and E. Santos, Wigner density of a rigid rotator, Phys. Rev. E 55 2551-2556 (1997).

[12] E. P. Wigner, On the quantum correction for thermodynamic equilibrium, Phys. Rev. 40749 (1932).

[13] T. Fischer, C. Gneiting and K. Hornberger, Wigner function for the orientation state, New Journ. Phys. 15063004 (10pp) (2013).

[14] M. Grigorescu, Classical probability waves, Physica A 3876497 (2008).

[15] M. Grigorescu, Functional coherent distributions on granular phasespace and quantization, research proposal 338620ERC-2013-ADG/Nov $22(2012)$.

[16] M. Grigorescu, Coherent distributions and quantization, e-print arXiv: 1410.1338 (2014).

[17] E. P. Wigner, Group Theory and its Applications to the Quantum Mechanics of the Atomic Spectra, Academic Press NY (1959).

[18] A. Sommerfeld, Thermodynamik und Statistik, Akademische Verlagsgesellschaft, Leipzig (1962).

[19] F. Hirzebruch, Neue Topologische Methoden in der algebraischen Geometrie, Springer (1956).

[20] S. M. Barnett and D. T. Pegg, Quantum theory of rotation angles, Phys. Rev. A 413427 (1990).

[21] D. Loss and K. Müllen, Commutation relations for periodic operators, J. Phys. A: Math. Gen. 25 L235 (1992).

[22] M. Naïmark and A. Stern, Théorie des Représentations des Groupes, Éditions Mir, Moscou (1979), p. 341.

[23] J. J. Slawianowski, V. Kovalchuck, A. Martens, B. Golubowska and E. Rozko, Quasiclassical and quantum systems of angular momentum. Part III: Group algebra $\mathfrak{s u}(2)$, quantum angular momentum and quasiclassical asymptotics, J. Geom. Sym. Phys. 23 59-95 (2011). 